\title{
Impact of probiotic feeding during weaning on the serum lipid profile and plasma metabolome in infants
}

\author{
Elin Chorell ${ }^{1} \dagger$, Frida Karlsson Videhult ${ }^{2} \dagger$, Olle Hernell ${ }^{2}$, Henrik Antti ${ }^{1 *} \ddagger$ and Christina E. West ${ }^{2 *} \ddagger$ \\ ${ }^{1}$ Department of Chemistry, Computational Life Science Cluster (CLiC), Umeå University, SE-901 85 Umeå, Sweden \\ ${ }^{2}$ Department of Clinical Sciences, Paediatrics, Faculty of Medicine, Umeå University, SE-901 85 Umeå, Sweden \\ (Submitted 16 March 2012 - Final revision received 6 September 2012 - Accepted 7 September 2012 - First published online 11 December 2012)
}

\begin{abstract}
The gut microbiome interacts with the host in the metabolic response to diet, and early microbial aberrancies may be linked to the development of obesity and metabolic disorders later in life. Probiotics have been proposed to affect metabolic programming and blood lipid levels, although studies are lacking in infants. Here, we report on the lipid profile and global metabolic response following daily feeding of probiotics during weaning. A total of 179 healthy, term infants were randomised to daily intake of cereals with ( $n$ 89) or without $(n 90)$ the addition of Lactobacillus paracasei ssp. paracasei F19 (LF19) $10^{8}$ colony-forming units per serving from 4 to 13 months of age. Weight, length and skinfold thickness were monitored. Venous blood was drawn at 5.5 and 13 months of age for analysis of the serum lipid profile. In a subsample, randomly selected from each group, GC-time-of-flight/MS was used to metabolically characterise plasma samples from thirty-seven infants. A combination of multi- and univariate analysis was applied to reveal differences related to LF19 treatment based on 228 putative metabolites, of which ninety-nine were identified or classified. We observed no effects of probiotic feeding on anthropometrics or the serum lipid profile. However, we detected significantly lower levels of palmitoleic acid $(16: 1)(P<0 \cdot 05)$ and significantly higher levels of putrescine $(P<0.01)$ in LF19-treated infants. Palmitoleic acid is a major MUFA strongly linked to visceral obesity, while putrescine is a polyamine with importance for gut integrity. Whether the observed differences will have long-term health consequences are being followed.
\end{abstract}

Key words: Probiotics: Lactobacillus paracasei ssp. paracasei strain F19: Metabolomics: Infant feeding

Early programming refers to prenatal and early postnatal factors that may influence the risk of developing chronic disease in adulthood ${ }^{(1)}$. Emerging evidence suggests that early life growth patterns play an important role in the aetiology of obesity $^{(2)}$. Infant feeding mode such as early introduction of solid foods and formula feeding seems to affect the risk of developing obesity ${ }^{(3)}$, although results are conflicting ${ }^{(4)}$ Breastfeeding has been proposed to decrease the risk of obesity and to lower serum cholesterol levels in adulthood ${ }^{(5)}$. Besides diet, another environmental exposure implicated in obesity development and associated metabolic disorders is the gut microbiota $^{(6)}$. At birth, the infant colon is rapidly colonised by microbes ${ }^{(7)}$. During weaning, there is increasing diversity of gut microbiota, but it takes years before the commensal microbiota is established. Gut microbiota can make an impact on metabolism by fermentation of nutrients and secretion of bioactive compounds ${ }^{(8)}$, and may exert effects on host gene expression in the enterohepatic system, including genes involved in metabolism and immune function. A disturbed gut microbiome, with increased numbers of Firmicutes and decreased numbers of Bacteroidetes, was demonstrated to be linked to energy harvest and obesity ${ }^{(9)}$, first in animal models and later also in human subjects ${ }^{(10-12)}$. This was followed by a report on aberrancies in infant gut microbiota preceding the development of obesity ${ }^{(13)}$, thereby proposing a possibility for prevention of obesity and associated metabolic disorders by modulation of gut microbiota.

Probiotics, live micro-organisms which when administered in adequate amounts confer a health benefit on the host ${ }^{(14)}$ have been proposed to affect metabolic programming ${ }^{(15,16)}$ and blood lipid concentrations. Animal studies have shown that the probiotic Lactobacillus paracasei ssp. paracasei

\footnotetext{
Abbreviations: HMCR, hierarchical multivariate curve resolution; LF19, Lactobacillus paracasei ssp. paracasei F19; OPLS-DA, orthogonal partial least squares discriminant analysis; PA, pipecolic acid; TC, total cholesterol.

*Corresponding authors: Dr H. Antti, fax +46 9078699 95, email henrik.antti@chem.umu.se; Dr C. E. West, fax +46 90 12 3728 , email christina.west@pediatri.umu.se

$\dagger$ These authors contributed equally to the present work.

‡ Shared senior authorship.
} 
strain F19 (LF19) induced up-regulation of genes involved in regulation of energy homeostasis ${ }^{(17)}$, reduced body fat and affected the lipoprotein profile ${ }^{(18)}$. Collectively, these studies suggest that LF19 may influence body composition as well as metabolic programming. However, whether these findings can be extrapolated to human subjects need to be verified.

The methodology of studying biochemical processes in biofluids and tissues, based on concentration changes of low molecular compounds, i.e. metabolites, is commonly known as metabolomics ${ }^{(19)}$. Metabolomics involves a combination of sensitive analytical techniques for metabolite detection and quantification and bioinformatics based on multivariate statistics for data analysis and evaluation. The methodology has been demonstrated as effective in screening for biomarkers or biomarker patterns and for understanding the underlying mechanisms of different biochemical processes ${ }^{(20,21)}$. Due to the close relationship among nutrients, gene transcription, gene expression and ultimately metabolism, the interest for metabolomics as a tool in nutritional research has rapidly increased. This also includes studying the effects of gut microbiota and microbial manipulation by probiotics on the human phenotype and ultimately how this can be utilised in the development towards personalised disease prevention by means of dietary modifications ${ }^{(22)}$.

Therefore, we explored the effects of supplementation with LF19 during weaning on the serum lipid profile and global plasma metabolome.

\section{Methods}

\section{Subjects}

The present study is part of a double-blind, placebocontrolled, randomised intervention trial on the effects of feeding the probiotic LF19 from 4 to 13 months of age. The study was registered at www.clinicaltrials.gov (NCT 00894816). The design has previously been described in detail $^{(23)}$. Briefly, the trial was conducted from August 2000 to November 2003. Inclusion criteria were healthy infants born at term, vaginally delivered with a birth weight $>2500 \mathrm{~g}$, no atopic manifestation or medication that could have affected gut microbiota, i.e. antibiotics or prior pre- or probiotic intake. Written and oral information was provided before enrolment, and written consent was signed by the parents. Infants were randomised to daily intake of cereals with or without the addition of $1 \times 10^{8}$ colony-forming units of strain LF19. The cereals contained cows' milk protein and were rice-based from 4 to 6 and wheat-based from 6 to 13 months of age. The composition of cereals is displayed in Table 1. The recommended intake was at least one serving of cereals daily from 4 to 13 months of age. Mean daily intake was 0.8 ( $\mathrm{SD} 0.5$ ) and 0.9 (SD 0.4 ) servings daily in the LF19 and placebo groups ${ }^{(23)}$, respectively. Parents were asked to abstain from feeding the infant other probioticcontaining products. No other dietary advice was given. Breastfeeding duration, intake of cereals and intake of other probiotics were prospectively followed by use of diary cards $^{(23)}$. Of 179 included infants, 171 completed the intervention; eighty-four and eighty-seven infants in the LF19 and placebo groups, respectively. The study was conducted following the World Medical Association Declaration of Helsinki ${ }^{(24)}$. Approval of the trial was obtained from the ethics committee of the Medical Faculty of Umeå University.

\section{Anthropometric data and blood samplings}

Weight, length, head circumference, knee-heel length and skinfold thickness were assessed at 4 (entry), 5.5, 6.5, 9, 12 and 13 months of age. Infants were weighed to the nearest $0.01 \mathrm{~kg}$ using a Seca 835 digital baby scale (Seca), and recumbent length was measured to the nearest $\mathrm{mm}$ using a Harpenden infantometer (CMS Weighing Equipment). Head circumference was measured using a non-elastic tape measure to the nearest $\mathrm{mm}$. Knee-heel length was measured using an infant knemometer (Infant Knemometer BK5, FORCE Instituttet). Triceps, biceps, suprailiac and subscapular skinfold thicknesses were measured using a standard skinfold caliper (Harpenden skinfold caliper HSK-BI, British Indicators), which was operated with a constant pressure of $10 \mathrm{~g} / \mathrm{mm}^{2}$.

Venous blood samples were collected at 5.5 and 13 months of age. Sera were stored at $-20^{\circ} \mathrm{C}$ until analysis. Plasma was stored at $-70^{\circ} \mathrm{C}$ until analysis.

\section{Biochemical analyses}

In total, serum samples were available from eighty-two and eighty-five infants in the LF19 and placebo groups, respectively. Of these, serum samples drawn at 5.5 months of age were available from sixty-eight and sixty-seven infants in the LF19 and placebo groups, respectively. The corresponding number at 13 months of age was seventy-three in both groups. Serum lipids were analysed for total cholesterol (TC), HDL-cholesterol, ApoA1, ApoB and TAG by the Roche/Hitachi method, which is the routine analysis used at the Department of Clinical Chemistry at Umeå University hospital. LDL-cholesterol was calculated using the Friedewald formula ${ }^{(25)}$.

\section{Metabolomic analysis}

For characterisation of the plasma metabolome, twenty infants from each treatment group were randomly selected.

\section{Sample preparation for GC-time-of-flight/MS analysis}

Frozen plasma samples were allowed to thaw at $37^{\circ} \mathrm{C}$ for $15 \mathrm{~min}$, and immediately thereafter $900 \mu \mathrm{l}$ of extraction solution (methanol-water in the ratio 8:1 containing eleven internal standards (each $7 \mathrm{ng} / \mu \mathrm{l})$ ) was added to a $100 \mu \mathrm{l}$ aliquot of plasma followed by vortexing for approximately $10 \mathrm{~s}$. After vortexing, the samples were vigorously shaken at $30 \mathrm{~Hz}$ for $1 \mathrm{~min}$ in a MM301 Vibration Mill (Retsch $\mathrm{GmbH}$ \& Company KG), then placed on ice for $120 \mathrm{~min}$ and subsequently centrifuged at $19600 \mathrm{~g}$ for $10 \mathrm{~min}$ at $4^{\circ} \mathrm{C}$. A $100 \mu \mathrm{l}$ aliquot of the supernatant was transferred to a GC vial and evaporated to dryness. Methoxymation using $15 \mu \mathrm{l}$ of methoxyamine solution in pyridine $(15 \mu \mathrm{g} / \mu \mathrm{l})$ was carried 
Table 1. Nutrient content of the study cereals

\begin{tabular}{|c|c|c|c|c|c|c|}
\hline & \multicolumn{2}{|c|}{ 4-6 months ${ }^{*}$} & \multicolumn{2}{|c|}{ 6-8 months $†$} & \multicolumn{2}{|c|}{ 8-13 months $\ddagger$} \\
\hline & Per $100 \mathrm{~g}$ & Per (serving $33 \mathrm{~g}$ )§ & Per $100 \mathrm{~g}$ & Per (serving $33 \mathrm{~g}$ )§ & Per $100 \mathrm{~g}$ & Per (serving $33 \mathrm{~g}) \S$ \\
\hline Energy (kJ) & 1950 & 650 & 1950 & 650 & 1900 & 600 \\
\hline Energy (kcal) & 470 & 150 & 460 & 150 & 450 & 150 \\
\hline Protein $(\mathrm{g})$ & $13 \cdot 0$ & 4.3 & 13.0 & $4 \cdot 3$ & $14 \cdot 0$ & 4.6 \\
\hline Carbohydrates (g) & $59 \cdot 0$ & $19 \cdot 5$ & $61 \cdot 0$ & $20 \cdot 0$ & $58 \cdot 0$ & $19 \cdot 0$ \\
\hline Sugars (g) & $30 \cdot 0$ & $9 \cdot 9$ & $26 \cdot 0$ & $8 \cdot 6$ & $25 \cdot 0$ & 8.5 \\
\hline Fat $(\mathrm{g})$ & $20 \cdot 0$ & $6 \cdot 6$ & $18 \cdot 0$ & 5.9 & $18 \cdot 0$ & 5.9 \\
\hline Saturated (g) & $6 \cdot 8$ & $2 \cdot 2$ & $6 \cdot 2$ & $2 \cdot 0$ & $6 \cdot 2$ & $2 \cdot 0$ \\
\hline Monounsaturated (g) & 8.6 & $2 \cdot 9$ & $7 \cdot 7$ & 2.5 & $7 \cdot 7$ & 2.5 \\
\hline Polyunsaturated $(\mathrm{g})$ & 4.6 & 1.5 & $4 \cdot 1$ & 1.4 & $4 \cdot 1$ & 1.4 \\
\hline Fibre $(\mathrm{g})$ & $2 \cdot 0$ & 0.7 & $2 \cdot 0$ & 0.7 & 4.0 & 1.3 \\
\hline Vitamin A $(\mu \mathrm{g})$ & 350 & 116 & 350 & 115 & 350 & 115 \\
\hline Vitamin $D(\mu \mathrm{g})$ & 8.5 & $2 \cdot 8$ & 8.5 & $2 \cdot 8$ & 8.5 & $2 \cdot 8$ \\
\hline Vitamin $E(\mathrm{mg})$ & $7 \cdot 0$ & $2 \cdot 3$ & $7 \cdot 0$ & $2 \cdot 3$ & $7 \cdot 0$ & $2 \cdot 3$ \\
\hline Vitamin C (mg) & 80 & 26 & 80 & 25 & 80 & 26 \\
\hline Thiamin (mg) & 0.7 & 0.2 & 0.7 & 0.2 & 0.7 & 0.2 \\
\hline Riboflavin (mg) & 0.5 & 0.2 & 0.5 & 0.2 & 0.5 & 0.2 \\
\hline Niacin (mg) & 3.5 & $1 \cdot 2$ & 3.5 & $1 \cdot 2$ & 3.5 & $1 \cdot 2$ \\
\hline Vitamin $B_{6}(\mathrm{mg})$ & 0.4 & 0.1 & 0.4 & 0.1 & 0.4 & $0 \cdot 1$ \\
\hline Folic acid $(\mu \mathrm{g})$ & 45 & 15 & 45 & 15 & 45 & 15 \\
\hline Vitamin $B_{12}(\mu \mathrm{g})$ & 1.0 & 0.3 & 1.0 & 0.3 & 1.0 & 0.3 \\
\hline Pantothenic acid (mg) & $2 \cdot 5$ & 0.8 & $2 \cdot 0$ & 0.7 & $2 \cdot 0$ & 0.7 \\
\hline $\mathrm{Cl}(\mathrm{mg})$ & 100 & 33 & 100 & 33 & 100 & 33 \\
\hline $\mathrm{Na}(\mathrm{mg})$ & 150 & 50 & 120 & 40 & 110 & 36 \\
\hline $\mathrm{K}(\mathrm{mg})$ & 850 & 280 & 640 & 211 & 800 & 264 \\
\hline Chloride (mg) & 350 & 116 & 270 & 89 & 270 & 89 \\
\hline $\mathrm{Ca}(\mathrm{mg})$ & 600 & 198 & 620 & 205 & 600 & 198 \\
\hline$P(m g)$ & 350 & 116 & 320 & 106 & 400 & 132 \\
\hline $\mathrm{Fe}(\mathrm{mg})$ & 8.5 & $2 \cdot 8$ & 8.5 & $2 \cdot 8$ & 8.5 & $2 \cdot 8$ \\
\hline $\mathrm{Mg}(\mathrm{mg})$ & 70 & 23 & 50 & 17 & 80 & 26 \\
\hline $\mathrm{Zn}(\mathrm{mg})$ & 1.5 & 0.5 & 1.5 & 0.5 & $2 \cdot 0$ & 0.7 \\
\hline lodine $(\mu \mathrm{g})$ & 50 & 17 & 50 & 17 & 50 & 17 \\
\hline
\end{tabular}

*Ingredients: rice starch, skimmed milk powder, vegetable oil (palm, rapeseed or sunflower), pineapple juice concentrate (16\%), banana powder (8\%), unsalted whey powder, oat starch, wheat starch, milk protein, emulsifier (vegetable mono- and diacylglycerols), vitamins and minerals, as indicated above.

† Ingredients: skimmed milk powder, rice starch, wheat starch, vegetable oil (palm, rapeseed and sunflower), apple juice concentrate (14\%), pear juice concentrate $(7 \%)$, dried apple $(4.5 \%)$, milk protein, emulsifier (vegetable mono- and diacylglycerols), vitamins and minerals, as indicated above.

¥Ingredients: skimmed milk powder, graham starch, vegetable oil (palm, rapeseed, sunflower), pear juice concentrate (12\%), rice starch, oat starch, banana powder ( $9.5 \%$ ), dried plum (3.5\%), milk protein), emulsifier (vegetable mono- and diacylglycerols), vitamins and minerals as indicated above.

$\S$ One serving of cereals contained $10^{8}$ colony-forming units of Lactobacillus paracasei ssp. paracasei $\mathrm{F} 19$.

out at room temperature for $16 \mathrm{~h}$. Finally, the sample was trimethylsilylated with $15 \mu \mathrm{l}$ of $N$-methyl- $N$-trimethylsilyltrifluoroacetamide at room temperature for $1 \mathrm{~h}$, after which $15 \mu \mathrm{l}$ of heptane (containing $0.5 \mu \mathrm{g}$ of methyl stearate as injection standard) was added.

\section{GC-time-of-flight/MS analysis}

Using a CTC Combi Pal autosampler (CTC Analytics AG), $1 \mu \mathrm{l}$ aliquot of derivatised sample was injected (splitless mode) into an Agilent 6980 GC equipped with a $10 \mathrm{~m} \times 0.18 \mathrm{~mm}$ inner diameter fused-silica capillary column chemically bonded with $0 \cdot 18 \mu \mathrm{m}$ DB5-MS stationary phase (J\&W Scientific). The injector temperature was set at $270^{\circ} \mathrm{C}$, and helium was used as the carrier gas at a constant flow rate of $1 \mathrm{ml} / \mathrm{min}$. In each analysis, the purge time was set to $60 \mathrm{~s}$ at a purge flow rate of $20 \mathrm{ml} / \mathrm{min}$ and equilibration time of $1 \mathrm{~min}$. The column temperature was initially kept at $70^{\circ} \mathrm{C}$ for $2 \mathrm{~min}$ and then increased by $30^{\circ} \mathrm{C} / \mathrm{min}$ to $320^{\circ} \mathrm{C}$, and held at that temperature for $2 \mathrm{~min}$. The column effluent was introduced into the ion source of a Pegasus III TOF/MS instrument
(Leco Corporation). The temperature was set at $250^{\circ} \mathrm{C}$ for the transfer line and at $200^{\circ} \mathrm{C}$ for the ion source. Ions were generated by a $70 \mathrm{eV}$ electron beam at a current of $2.0 \mathrm{~mA}$. Masses were acquired from $\mathrm{m} / z 50$ to 800 at a rate of $30 \mathrm{spectra} / \mathrm{s}$, and the acceleration voltage was turned on after a solvent delay of $165 \mathrm{~s}$. Files of acquired samples were exported to MATLAB 7.11.0.584 (R2010b) (Mathworks) in NetCDF (Network Common Data Form) format for further data processing and analysis.

\section{Hierarchical multivariate curve resolution}

All data pre-treatment procedures, including baseline correction, chromatogram alignment, setting of time windows and hierarchical multivariate curve resolution (HMCR), were performed in MATLAB (Mathworks) using in-house scripts. All samples (including repeated extractions) were subjected to HMCR. Alignment and smoothing using a moving average were carried out before HMCR was used to resolve pure chromatographic and spectral profiles. Before multivariate modelling, all peak areas were normalised to peak areas of 
eleven internal standards eluting over the entire chromatographic time range. Resolved mass spectral profiles were subjected to spectral database search by means of NIST library 2.0 (as of 31 January 2001), using the in-house mass spectra library database established by Umeå Plant Science Center or the mass spectra library maintained by the Max Planck Institute in Golm (http://csbdb.mpimp-golm.mpg.de/csbdb/ gmd/msri/gmd_contributions.html). Match values ranking the spectra were calculated using the dot product of the two spectra (i.e. the resolved spectrum and the database spectrum), with higher $m / z$ peaks having more weight than lower $m / z$ peaks, as higher $m / z$ values are considered to be more compound specific. The match values range from 0 to 999 , where 999 indicates an identical match. Positive identification was performed by combining match values with retention time index, calculated from the analytically characterised alkane series (C10-C40). Further, all metabolites were classified as amino acids, amines, alcohol/polyol, carbohydrates, fatty acids, carboxylic acids, hydroxyl acids, sterols, non-classified or unidentified. Assignments into different compound classes were based on the criteria from the human metabolome database ${ }^{(26)}$.

\section{Statistical analysis}

For analysis of demographic characteristics, $\chi^{2}$ tests for categorical data and Student's $t$ test for numerical data were used. Student's $t$ test was used to compare the two treatment groups. ANCOVA was used to assess serum lipid and apo levels with breastfeeding duration until commencement of the intervention and sex as covariates. Anthropometric data were analysed by longitudinal repeated measures of variance, adjusted for measurements at 4 months (entry) as a covariate. Only infants with measures from all six measurements were included. These statistical analyses were performed with SPSS software (version 18.0; SPSS, Inc.). Differences were considered significant at the level of $P<0.05$. The HMCR-processed GC-time-of-flight/MS data were mean-centred and scaled to unit variance prior to any multivariate data analysis. Principal component analysis was utilised for data overview and to detect clusters or outliers. Orthogonal partial least squares discriminant analysis (OPLS-DA) was performed to model the systematic variation in the metabolomics data related and orthogonal to pre-defined sample classes among the plasma samples here, considering, placebo $v$. LF19 treatment and 5.5 v. 13 months of age. Cross-validation was used to determine the predictive ability of the models, and ANOVA based on the cross-validated OPLS-DA score vectors (CV-ANOVA) was employed to calculate $P$ values for the differences between the pre-defined sample classes in the respective models. In the case of modelling the differences between samples collected at $5.5 v .13$ months of age, including both treatment groups, a test set of eight samples (four from each group) was selected to test the predictive ability of the model. The OPLS-DA model loadings, combined with univariate $P$ values (Mann-Whitney), were used to highlight significant metabolites associated with detected class differences. All multivariate analyses were carried out using the SIMCA-P+ software (version 12.0.1, Umetrics $\mathrm{AB}$ ) and

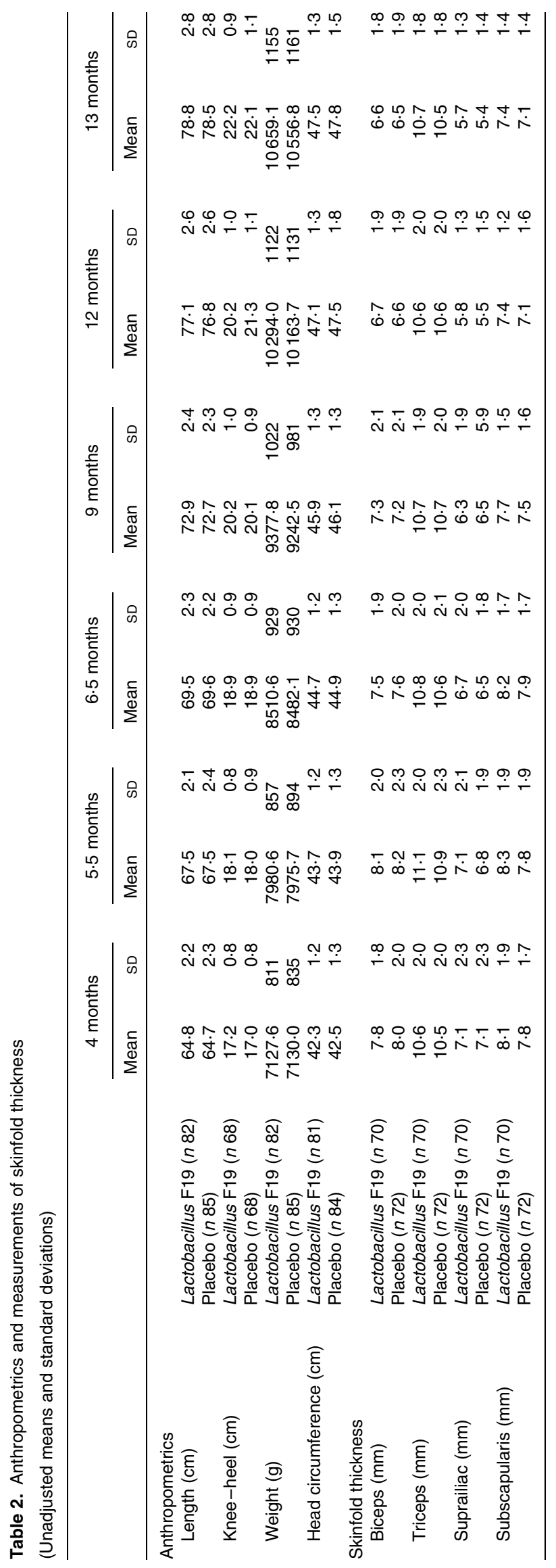


Mann-Whitney test was performed using R software (version 2.11.1, R Foundation for Statistical Computing).

\section{Results}

\section{Study population}

There was no difference between the two groups in distribution of boys and girls; 39 and $45 \%$ boys were included in the LF19 and placebo groups, respectively $(P>0 \cdot 05)$. Mean gestational age was 40.3 (SD 1.1) and 39.9 (SD 1.3) weeks in the LF19 and placebo groups, respectively $(P>0.05)$. Mean exclusive breastfeeding duration was 3.8 (SD 1.2) months in both groups and mean total breastfeeding duration was $8 \cdot 2$ (SD 3.0) and 7.9 (SD 3.3) months in the LF19 and placebo groups, respectively $(P>0 \cdot 05)$. There were no differences between the two groups in these variables when the statistical analysis was restricted to the subgroup of infants included in plasma metabolome analysis (data not shown).

\section{Infant anthropometrics}

There was no difference in length, weight or skinfold thickness between the two groups at any age (Table 2).

\section{Serum lipids}

There were no differences in TAG, TC, HDL-cholesterol, LDL-cholesterol, apoA1 and apoB concentrations or apoA1: apoB ratio between the two groups at 5.5 months of age $(P>0.05)$ (Table 3). Adjusting for breastfeeding duration and sex did not affect the outcome (data not shown). At 13 months of age, there was a trend towards lower TC, LDL and apoA1 levels in the probiotic group, but these differences did not reach statistical significance (Table 3). Adjusting for breastfeeding duration and sex did not significantly affect the outcome (data not shown).

\section{Plasma metabolome}

In total, plasma samples from thirty-seven infants (nineteen from the LF19 and eighteen from the placebo group) from two different time points, 5.5 and 13 months of age, were metabolically characterised using GC/time-of-flight-MS. From these, three samples were excluded due to low analytical quality. The collected GC/time-of-flight-MS data from the remaining seventy-one samples were subjected to HMCR for data processing. This resulted in 304 potential compound peaks. After excluding peaks originating from internal standards, contaminations and low quality spectra, 228 putative metabolites remained from which ninety-nine were successfully identified or classified (Table 4). The rest of the compounds were not identified with enough certainty, using mass spectral library search, but still retained information regarding retention time index and fragmentation patterns.

\section{Effects of Lactobacillus paracasei ssp. paracasei F19}

No significant difference was found in plasma metabolite profile between 5.5-month-old infants in relation to LF19 treatment. Furthermore, we could not obtain a significant multivariate model for the difference in plasma metabolite profiles between 13-month-old infants in relation to LF19 treatment, i.e. comparing infants fed LF19 with placebo-fed infants. However, there were still unique metabolites revealing significant differences between the two groups.

Compared with the placebo, the LF19 group had significantly lower levels $(P<0 \cdot 04)$ of the SFA, palmitic acid $(16: 0)$ and the MUFA palmitoleic acid $(16: 1)$. Additionally, we observed significantly higher levels $(P<0.01)$ of tryptophan, a branched-chain amino acid, and putrescine, a growth factor polyamine important for cell division, in the LF19 compared with the placebo group at 13 months of age. There were also significantly higher levels $(P=0.02)$ of D-methyl-glucopyranoside ( $\beta$-D-glucose) in the LF19 compared with the placebo group.

A robust and significant metabolite pattern $(P<0.001)$ was obtained for the difference in plasma profiles between infants at 5.5 and 13 months of age in both treatment groups. The OPLS-DA model, including samples from both treatment groups, revealed a classification accuracy of $96 \%$ based on the model fit and $95 \%$ based on independent sample predictions (Fig. 1).

To further investigate the influence of LF19 treatment on metabolic response during the second half of infancy, separate

Table 3. Serum lipid and apolipoprotein levels in infants at 5.5 and 13 months of age (Mean values and standard deviations)

\begin{tabular}{|c|c|c|c|c|c|c|c|c|c|c|}
\hline & \multicolumn{5}{|c|}{5.5 months } & \multicolumn{5}{|c|}{13 months } \\
\hline & \multicolumn{2}{|c|}{$\begin{array}{l}\text { Lactobacillus } \\
\text { F19 }(n 68)\end{array}$} & \multicolumn{2}{|c|}{ Placebo ( $n 67)$} & \multirow[b]{2}{*}{$P$} & \multicolumn{2}{|c|}{$\begin{array}{l}\text { Lactobacillus } \\
\text { F19 }(n 73)\end{array}$} & \multicolumn{2}{|c|}{ Placebo ( $n 73)$} & \multirow[b]{2}{*}{$P$} \\
\hline & Mean & SD & Mean & SD & & Mean & SD & Mean & SD & \\
\hline TC $(\mathrm{mmol} / \mathrm{l})$ & 3.78 & 0.73 & 3.70 & 0.75 & 0.54 & 3.60 & 0.61 & $3 \cdot 76$ & 0.59 & 0.11 \\
\hline TAG (mmol/l) & 1.92 & 0.83 & $2 \cdot 01^{*}$ & 0.83 & 0.52 & 1.65 & 0.73 & 1.59 & 0.70 & 0.63 \\
\hline HDL-C (mmol/l) & 1.07 & 0.22 & 1.01 & 0.25 & 0.16 & 0.99 & 0.25 & 1.03 & 0.21 & 0.28 \\
\hline LDL-C (mmol/l) & 1.85 & 0.79 & $1 \cdot 77^{*}$ & 0.74 & 0.58 & 1.87 & 0.59 & $2 \cdot 01$ & 0.55 & 0.14 \\
\hline ApoA1 (g/l) & 1.06 & 0.14 & 1.03 & 0.16 & 0.29 & 0.97 & 0.17 & 1.01 & 0.16 & 0.11 \\
\hline ApoB $(\mathrm{g} / \mathrm{l})$ & 0.72 & 0.19 & 0.73 & 0.20 & 0.76 & 0.73 & 0.14 & 0.75 & 0.13 & 0.30 \\
\hline ApoB:ApoA1 & 0.69 & 0.20 & 0.73 & 0.24 & 0.33 & 0.78 & 0.23 & 0.76 & 0.18 & 0.62 \\
\hline
\end{tabular}

TC, total cholesterol; HDL-C, HDL-cholesterol; LDL-C, LDL-cholesterol. ${ }^{\star}$ Data missing from one individual. 
NS British Journal of Nutrition

Table 4. Identified and classified metabolites

\begin{tabular}{|c|c|c|c|c|c|c|c|c|}
\hline $\begin{array}{l}\text { Number } \\
\text { in plot }\end{array}$ & Compound class & Identification & Abbreviation & $\begin{array}{l}\text { Placebo } v \text {. LF19 } \\
\text { at } 13 \text { months } \\
\text { (response in LF19) }\end{array}$ & $\begin{array}{l}\text { Age response } \\
\text { in placebo }\end{array}$ & $\begin{array}{l}\text { Age response } \\
\text { in LF19 }\end{array}$ & $\begin{array}{c}\text { Match } \\
\text { value†‡ }\end{array}$ & $\mathrm{RI}-\mathrm{RI}_{\text {Library }} \S$ \\
\hline 5 & Hydroxy acid & Lactic acid & LacA & & $\uparrow$ & $\downarrow$ & 888 & 30 \\
\hline 9 & Hydroxy acid & $\alpha$-Hydroxy acid & $\mathrm{AHA}$ & & $\uparrow^{*}$ & $\uparrow$ & 800 & 31 \\
\hline 14 & Amino acid & Alanine & Ala & & $\uparrow$ & $\downarrow$ & 894 & 32 \\
\hline 20 & Carboxylic acid & Oxalic acid & OxA & & $\uparrow$ & $\downarrow^{*}$ & 752 & 25 \\
\hline 25 & Fatty acid & Butyric acid $(4: 0)$ & BuA & & $\downarrow$ & $\downarrow$ * & 882 & - \\
\hline 26 & Fatty acid & Heptanoic acid $(7: 0)$ & НерА & & $\uparrow$ & $\downarrow$ & 874 & 7 \\
\hline 34 & Amino acid & - & & & $\downarrow$ & $\downarrow$ & 722 & 2 \\
\hline 38 & Carboxylic acid & Ketoleucine & & & $\downarrow$ & $\downarrow$ & 721 & 6 \\
\hline 39 & Amino acid & Valine & Val & & $\uparrow^{*}$ & $\uparrow^{*}$ & 883 & 3 \\
\hline 42 & Amine & Ethanolamine & EA & & $\uparrow$ & $\downarrow$ & 818 & 5 \\
\hline 44 & Amine & Urea & & & $\uparrow^{*}$ & $\uparrow^{*}$ & 823 & 6 \\
\hline 47 & Alcohol/polyol & Glycerol & & & $\downarrow$ & $\downarrow$ & 843 & 0 \\
\hline 48 & No class & Phosphoric acid & $\mathrm{PhA}$ & & $\uparrow^{*}$ & $\uparrow$ & 946 & 1 \\
\hline 50 & Amino acid & Isoleucine & Ile & & $\uparrow *$ & $\uparrow$ & 947 & 1 \\
\hline 52 & Amino acid & Proline & Pro & & $\uparrow *$ & $\uparrow$ & 894 & 5 \\
\hline 54 & Amino acid & Glycine & Gly & & $\uparrow *$ & $\uparrow^{*}$ & 954 & 2 \\
\hline 57 & Carboxylic acid & Succinic acid & & & $\uparrow$ & $\uparrow$ & 883 & 0 \\
\hline 58 & Hydroxy acid & Glycerate & GlyA & & $\uparrow^{*}$ & $\downarrow^{*}$ & 973 & 4 \\
\hline 59 & Amino acid & Pipecolic acid & PA & & $\uparrow^{*}$ & $\uparrow^{*}$ & 831 & 35 \\
\hline 65 & Amino acid & Serine & Ser & & $\uparrow^{*}$ & $\uparrow$ & 888 & 2 \\
\hline 70 & Amino acid & Threonine & Thr & & $\uparrow$ & $\downarrow$ & 882 & 2 \\
\hline 74 & Carboxylic acid & Glutaric acid & Glutarate & & $\uparrow^{*}$ & $\uparrow^{*}$ & 864 & 4 \\
\hline 81 & Amino acid & Methylcysteine & MeCys & & $\downarrow$ & $\downarrow^{*}$ & 869 & 7 \\
\hline 83 & Amino acid & $\beta$-Alanine & bAla & & $\downarrow^{*}$ & $\downarrow$ * & 907 & 4 \\
\hline 92 & Carbohydrate & Erythrose & & & $\uparrow$ & $\downarrow$ & 631 & 4 \\
\hline 93 & Alcohol/polyol & Erythritol & & & $\downarrow$ & $\downarrow^{*}$ & 760 & 0 \\
\hline 94 & Amino acid & Methionine & Met & & $\uparrow$ & $\uparrow$ & 884 & 0 \\
\hline 95 & Amino acid & Aspartic acid & AspA & & $\uparrow^{*}$ & $\uparrow^{*}$ & 943 & 2 \\
\hline 96 & Amino acid & Pyroglutamic acid & PyrA & & $\uparrow *$ & $\downarrow$ & 953 & 0 \\
\hline 97 & Amino acid & Hydroxyproline & $\mathrm{OH}-\mathrm{Pro}$ & & $\downarrow$ & $\downarrow$ * & 803 & 1 \\
\hline 99 & Hydroxy acid & Erythronic acid/threonic acid & & & $\downarrow$ & $\downarrow$ & 918 & 1 \\
\hline 100 & No class & Boric acid & & & $\uparrow$ & $\downarrow$ & 708 & 1 \\
\hline 103 & No class & Creatinine & $\mathrm{Cr}$ & & $\uparrow^{*}$ & $\uparrow$ & 849 & - \\
\hline 104 & Amino acid & Cysteine & Cys & & $\downarrow$ * & $\downarrow^{*}$ & 745 & 1 \\
\hline 105 & Hydroxy acid & Threonic acid & ThrA & & $\uparrow$ & $\downarrow$ * & 942 & 3 \\
\hline 108 & Amino acid & Ornithine & Orn & & $\uparrow$ & $\downarrow$ & 963 & 2 \\
\hline 109 & Amino acid & Glutamic acid & GlutA & & $\uparrow$ & $\uparrow$ & 890 & 2 \\
\hline 112 & Amino acid & Phenylalanine & Phe & & $\uparrow^{*}$ & $\uparrow^{*}$ & 962 & 0 \\
\hline 116 & Carbohydrate & Xylose & & & $\downarrow^{*}$ & $\downarrow^{*}$ & 741 & 2 \\
\hline 117 & Fatty acid & Lauric acid $(12: 0)$ & LauA & & $\downarrow$ * & $\downarrow$ * & 901 & 0 \\
\hline 118 & Carbohydrate & Arabinose & & & $\downarrow$ & $\downarrow$ * & 897 & 2 \\
\hline 119 & Amino acid & Asparagine & Asp & & $\uparrow$ & $\downarrow$ & 931 & 1 \\
\hline 120 & Carbohydrate & Ribose & & & $\uparrow$ & $\downarrow$ & 861 & 4 \\
\hline 124 & Amino acid & Lysine & Lys & & $\uparrow$ & $\downarrow$ & 826 & 3 \\
\hline 125 & Alcohol/polyol & Ribitol & & & $\uparrow$ & $\downarrow^{*}$ & 942 & 12 \\
\hline 126 & Carbohydrate & Rhamnose & & & $\downarrow^{*}$ & $\downarrow$ * & 790 & 2 \\
\hline 127 & Amino acid & Glutamine & Glu & & $\downarrow$ * & $\downarrow$ * & 918 & 3 \\
\hline 128 & Amine & Putrescine & Putr & $\uparrow^{*}$ & $\downarrow$ & $\downarrow$ * & 636 & 11 \\
\hline 132 & Alcohol/polyol & Glycerol-3-phosphate & & & $\uparrow$ & $\uparrow$ & 943 & 4 \\
\hline 135 & Carboxylic acid & Azelaic acid & $\mathrm{AzA}$ & & $\uparrow^{*}$ & $\uparrow^{*}$ & 867 & 4 \\
\hline
\end{tabular}


Table 4. Continued

\begin{tabular}{|c|c|c|c|c|c|c|c|c|}
\hline $\begin{array}{l}\text { Number } \\
\text { in plot }\end{array}$ & Compound class & Identification & Abbreviation & $\begin{array}{l}\text { Placebo } v \text {. LF19 } \\
\text { at } 13 \text { months } \\
\text { (response in LF19) }\end{array}$ & $\begin{array}{l}\text { Age response } \\
\text { in placebo }\end{array}$ & $\begin{array}{l}\text { Age response } \\
\text { in LF19 }\end{array}$ & $\begin{array}{c}\text { Match } \\
\text { value†‡ }\end{array}$ & $\mathrm{RI}-\mathrm{R} \mathrm{I}_{\text {Library }} \S$ \\
\hline 136 & No class & Hypoxanthine & Hxt & & $\downarrow$ & $\uparrow$ & 756 & 17 \\
\hline 137 & Carboxylic acid & Citric acid & $\mathrm{CA}$ & & $\downarrow$ & $\downarrow^{*}$ & 847 & 2 \\
\hline 139 & Amino acid & Arginine & $\operatorname{Arg}$ & & $\uparrow$ & $\uparrow$ & 935 & 0 \\
\hline 143 & Fatty acid & Myristic acid $(14: 0)$ & MyA & & $\downarrow^{*}$ & $\downarrow^{*}$ & 878 & 4 \\
\hline 145 & Carbohydrate & Fructose & & & $\uparrow *$ & $\uparrow$ & 957 & 1 \\
\hline 146 & Carbohydrate & Glucose & & & $\downarrow$ & $\downarrow^{*}$ & - & - \\
\hline 147 & Amino acid & Tyrosine & Tyr & & $\uparrow^{*}$ & $\downarrow$ & 914 & 6 \\
\hline 148 & Carbohydrate & D-Galactono-1,4-lactone & DGL & & $\uparrow$ & $\downarrow$ * & 929 & 14 \\
\hline 149 & Carbohydrate & Glucopyranose & & & $\uparrow$ & $\downarrow$ * & 919 & 7 \\
\hline 153 & Alcohol/polyol & myo-Inositol & & & $\downarrow$ * & $\downarrow$ * & 896 & 23 \\
\hline 154 & Carbohydrate & $\beta$-D-Methyl-glucopyranoside & Beta-D-glucose & $\downarrow$ * & $\downarrow$ & $\downarrow *$ & 956 & 0 \\
\hline 157 & Fatty acid & Palmitoleic acid $(16: 1)$ & PalA & $\downarrow$ * & $\downarrow$ & $\downarrow$ & 942 & 3 \\
\hline 159 & Fatty acid & Palmitic acid $(16: 0)$ & PA & $\downarrow$ * & $\uparrow$ & $\uparrow$ & 940 & 6 \\
\hline 164 & No class & Uric acid & UA & & $\uparrow$ & $\downarrow$ & 783 & 1 \\
\hline 165 & Carbohydrate & Sugar & & & $\uparrow$ & $\downarrow$ * & - & - \\
\hline 168 & Fatty acid & - & & & $\uparrow$ & $\uparrow$ & - & - \\
\hline 170 & Amino acid & Trypthophan & Trp & $\uparrow^{*}$ & $\uparrow$ & $\uparrow$ & 962 & 5 \\
\hline 171 & Fatty acid & Linoleic acid $(18: 2 n-6)$ & LA & & $\downarrow$ & $\uparrow$ & 855 & 2 \\
\hline 172 & Fatty acid & Oleic acid $(18: 1)$ & $\mathrm{OA}$ & & $\downarrow$ & $\downarrow$ & 956 & 2 \\
\hline 173 & Fatty acid & Stearic acid $(18: 0)$ & SA & & $\downarrow$ & $\downarrow$ & 968 & 1 \\
\hline 174 & Carbohydrate & Erythritol & & & $\downarrow$ & $\uparrow$ & 751 & 2 \\
\hline 175 & Amino acid & Cystine & oxCys & & $\downarrow$ & $\downarrow$ & 912 & 2 \\
\hline 176 & Fatty acid & & & & $\uparrow$ & $\uparrow$ & - & - \\
\hline 178 & Fatty acid & Arachidonic acid $(20: 4 n-6)$ & AA & & $\uparrow$ & $\downarrow$ & 916 & 1 \\
\hline 179 & Alcohol/polyol & myo-Inositol-1-phosphate & & & $\uparrow$ & $\downarrow$ & 934 & 0 \\
\hline 181 & Fatty acid & & & $\downarrow$ * & $\uparrow$ & $\downarrow$ & - & - \\
\hline 182 & Fatty acid & & & & $\uparrow$ & $\downarrow$ & - & - \\
\hline 185 & No class & Uridine & & & $\uparrow$ & $\uparrow$ & 751 & 16 \\
\hline 186 & Fatty acid & Fatty acid & & & $\downarrow$ & $\downarrow$ & - & - \\
\hline 188 & No class & Serotonine & & & $\uparrow$ & $\downarrow$ & 804 & 8 \\
\hline 196 & Fatty acid & Docosahexaenoic acid (22:6n-3) & DHA & & $\uparrow$ & $\uparrow$ & 885 & 2 \\
\hline 197 & No class & Inosine & & & $\uparrow$ & $\downarrow$ & 928 & 7 \\
\hline 198 & Fatty acid & - & & $\uparrow^{*}$ & $\uparrow$ & $\uparrow$ & - & - \\
\hline 199 & No class & Monohexadecanoylglycerol & & & $\uparrow$ & $\downarrow$ & 889 & 4 \\
\hline 202 & Carbohydrate & Lactose & & & $\uparrow$ & $\downarrow$ & 864 & 5 \\
\hline 203 & Carbohydrate & & & & $\uparrow$ & $\downarrow$ & - & - \\
\hline 204 & Carbohydrate & & & & $\uparrow$ & $\uparrow$ & - & - \\
\hline 205 & Carbohydrate & & & & $\uparrow$ & $\downarrow$ & - & - \\
\hline 206 & Carbohydrate & Maltose & & & $\uparrow$ & $\downarrow$ & 900 & 2 \\
\hline 209 & Sterol & - & & & $\downarrow$ & $\downarrow$ & - & - \\
\hline 210 & Sterol & - & & & $\uparrow$ & $\downarrow$ & - & - \\
\hline 216 & Alcohol/polyol & $\alpha$-Tocopherol & AT & & $\uparrow$ & $\downarrow$ & 919 & 2 \\
\hline 217 & Sterol & Cholesterol & Chol & & $\uparrow$ & $\downarrow$ & 903 & 8 \\
\hline 221 & Sterol & $\beta$-Sitosterol & & & $\downarrow$ & $\downarrow$ & 806 & 3 \\
\hline
\end{tabular}

LF19, Lactobacillus paracasei ssp. paracasei F19; RI, retention index; $\uparrow$, increase; $\downarrow$, decrease.

* Significant change $(P<0.05)$ comparing sample taken at 5.5 months of age with those taken at 13 months of age.

‡ Mass spectra searches were done with in-house libraries and the Max Planck Institute library in Golm (http://csbdb.mpimp-golm.mpg.de/csbdb/gmd/gmd.html).

$\S \mathrm{RI}$ calculated from analytically characterised alkane series (C10-C40). RI Library from mass spectra library database. 
OPLS-DA models were calculated for each treatment group in relation to age. Both groups revealed a significant separation $(P<0 \cdot 001)$ of plasma profiles comparing samples collected at 5.5 months with those collected at 13 months of age (Fig. 2). Since no independent sample prediction could be made due to a too low number of samples, the validation of class separations was made using cross-validation.

In both treatment groups, we observed a general change in amino acid profile during the second half of infancy. More specifically, there was a significant increase $(P<0.05)$ of pipecolic acid (PA), phenylalanine, aspartic acid, valine and glycine, while cysteine, glutamine and $\beta$-alanine decreased with age. Additionally, we detected a significant decrease $(P<0.001)$ in myo-inositol levels as well as in the SFA, lauric acid (12:0) and myristic acid (14:0) $(P<0 \cdot 04)$, with age (Fig. 2(c)).

By plotting the OPLS-DA correlation loadings for each treatment group against each other in a so-called shared and unique structure $\operatorname{plot}^{(27)}$, we could interpret the joint and opposite responses between the treatment groups (along the diagonals) as well as the unique response for each treatment group (along the axes) in relation to age (Fig. 2(c)).

Unique age-related metabolite changes in the LF19 group were seen, as a significant decrease in the levels of the SCFA butyric acid ( $4: 0)(P=0.02)$ and of methylcysteine $(P=0.01)$, in combination with a trend of decreased cholesterol, was detected in the model loadings. No such change was detected in the placebo group.

Another trend indicating differences between LF19 and placebo was an increase in the $n-6$ PUFA linoleic acid $(18: 2 n-6)$ in the LF19 group, whereas a reversed pattern was observed in the placebo group. In addition, we detected a decrease with age in metabolites linked to the pentose phosphate pathway in the LF19 group. This decrease was seen in erythrose, glycerate, D-galaculono-1,4-lactone and glucopyranose $(\beta$-D-glucose). Again, a reverse response was seen in the placebo group (Fig. 2(c)).

We could also detect individual differences in metabolic response from 5.5 to 13 months of age in both treatment groups. By subtracting the model score value for the 5.5-month sample from the corresponding 13-month sample, it became evident in the score plots (Fig. 2(a) and (b)) that

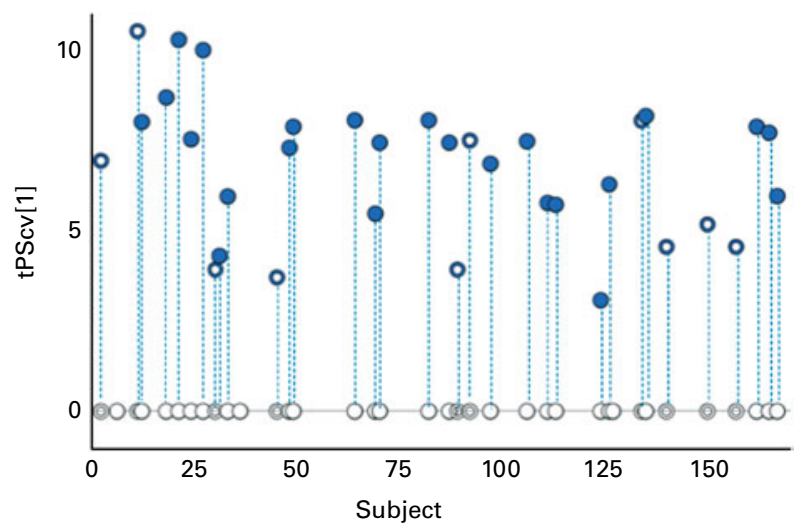

Fig. 1. Cross-validated orthogonal partial least squares discriminant analysis scores (tPScv[1]) revealing a clear difference in samples related to age. ๑, Model samples; ๑ independent samples predicted into the model. individual development varied among subjects irrespective of LF19 treatment.

\section{Discussion}

The aim of the present study was to elucidate whether feeding the probiotic LF19 during weaning would affect the lipid profile and global metabolome. The main findings of this study were significantly lower levels of the fatty acids palmitic (16:0) and palmitoleic acid (16:1) and significantly higher levels of tryptophan and putrescine in the LF19 compared with the placebo group at 13 months of age, while no significant effect by LF19 on the serum lipid profile was observed. Further, robust age-related metabolic patterns were observed in both treatment groups, with an increase in PA, phenylalanine, aspartic acid, valine and glycine, and a significant decrease in cysteine, glutamine and $\beta$-alanine during the second half of infancy.

Palmitoleic acid (16:1), one of the MUFA and an important product of endogenous lipogenesis, decreased in the LF19 group. In adults, plasma palmitoleic acid levels correlate strongly with weight, BMI, visceral adiposity, skinfold thickness ${ }^{(28)}$, hypertriacylglycerolaemia ${ }^{(29)}$ and have a significant relation to visceral adiposity in children ${ }^{(30)}$. The mechanisms linking MUFA content and visceral obesity need further clarification, but serum palmitoleic acid (16:1) levels reflect the hepatic lipid pool. It is proposed that elevated palmitoleic levels represent a shift of carbon from carbohydrates to fatty acids that may be linked to visceral obesity ${ }^{(31)}$. Previously, LF19 decreased fat storage in the abdominal and visceral regions in mice ${ }^{(18)}$. We observed no differences between the treatment groups in anthropometrics. Possibly, differences might have been detectable by the use of more sensitive measurements of visceral adiposity ${ }^{(32)}$. Alternatively, the observed effects in the murine model are not applicable to the more complex human setting.

Following 9 months of probiotic intake, there was a trend towards lower cholesterol levels compared with the placebo, but the difference was not statistically significant. A recent meta-analysis indicated that probiotics decrease TC and LDL levels in adults with both high and normal cholesterol levels ${ }^{(33)}$. The hypocholesterolaemic effects of probiotics have been attributed to deconjugation of bile acids, binding of cholesterol to cellular surfaces and production of SCFA ${ }^{(16)}$. As butyric acid was lower in the LF19 group, this cannot explain the trend towards lower cholesterol levels. The faecal pattern of SCFA reflects the functional status of the gut microbiota. Acetic, propionic and butyric acids are important for gut epithelial cell proliferation and differentiation ${ }^{(34)}$. In early infancy, production of acetic acid is predominant, followed by propionic and butyric acid. With age, acetic acid decreases while the other SCFA increase, reflecting the development of a more complex anaerobic microbiota ${ }^{(35)}$. All SCFA were not detected in plasma, but effects of LF19 on the faecal pattern of SCFA in the present study population will be reported elsewhere (CE West, E Norin, ML Hammarström, O Hernell, unpublished results). To the best of our knowledge, the present study is the first on probiotic effects on lipid profiles in infants. 

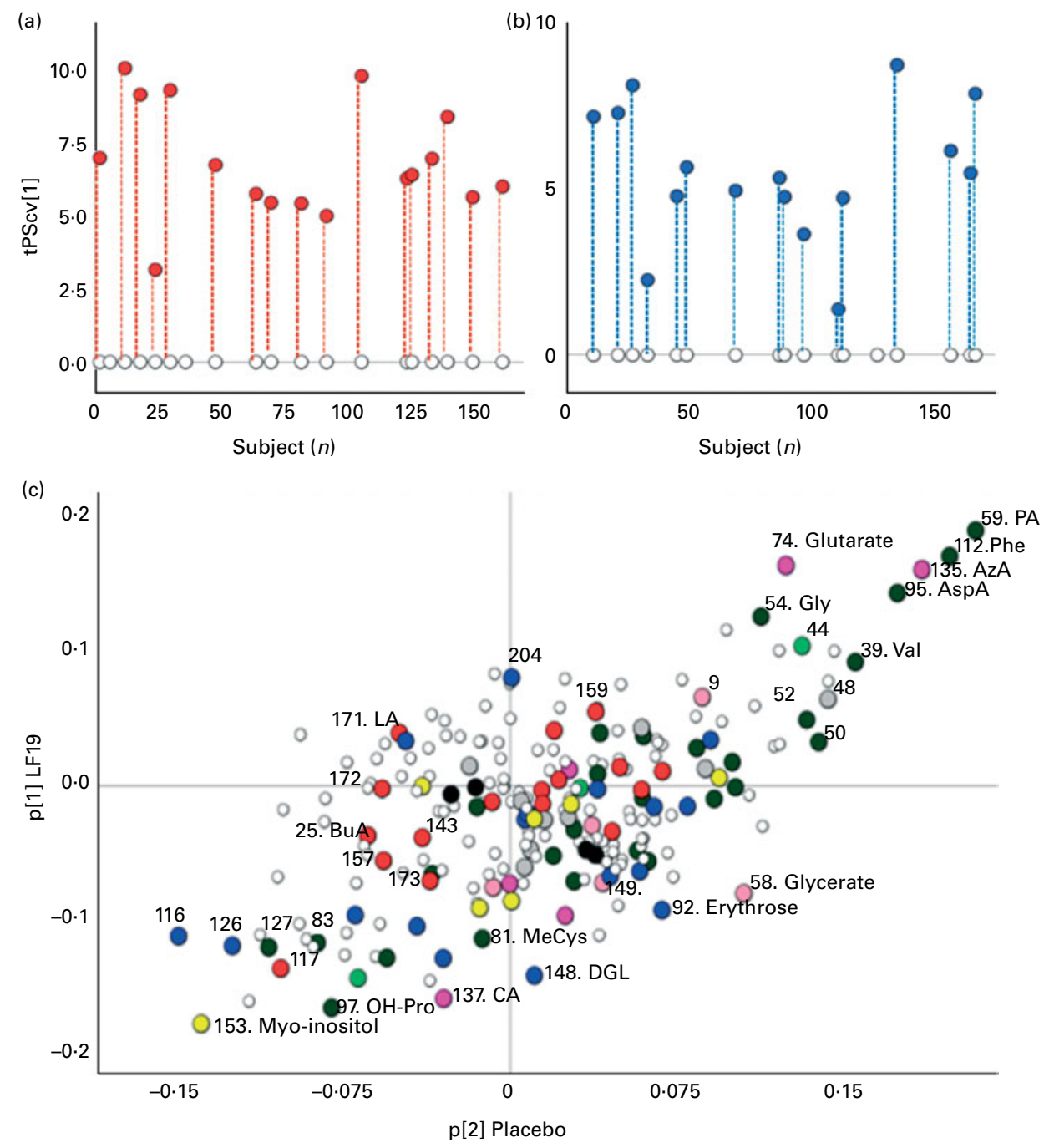

Fig. 2. Separate orthogonal partial least squares discriminant analysis (OPLS-DA) models for the Lactobacillus paracasei ssp. paracasei F19 (LF19) and placebo groups, comparing samples at 5.5 and 13 months of age. (a) Cross-validated scores describing a clear response with age for all samples in the LF19-treated infants. O, Samples collected at 5.5 months of age; O, samples collected at 13 months; each subject's corresponding samples are connected with ---. (b) Crossvalidated scores describing a clear separation of samples related to age in the placebo group. O, Samples collected at 5.5 months of age; O, samples collected at 13 months; each subject's corresponding samples are connected with ---. (c) Loadings (p[1]) from the two OPLS-DA models, LF19 or placebo, discriminating between samples taken at 5.5 and 13 months of age plotted against each other in a shared and unique structure plot. Joint and opposite metabolite changes for both groups are displayed along the diagonals, while group-specific changes are seen along the $x$ - (placebo) and $y$-axis (LF19). O, Identified metabolite; $\bullet$, sterol; ๑, amino acid; O, amine; O, alcohol/polyol; O, carbohydrate; O, fatty acid; O, carboxylic acid; O, hydroxyl acid and O, unidentified metabolite. PA, pipecolic acid; Phe, phenylalanine; AzA, azelaic acid; AspA, aspartic acid; Gly, glycine; Val, valine; MeCys, methylcysteine; DGL, D-galaculono-1,4-lactone; OH-Pro, hydroxyproline.

For comparison, there was no effect of galacto-oligosaccharides and long-chain fructo-oligosaccharides in infant formula on serum TC or TAG levels, compared with the control formula ${ }^{(36)}$. As lipids are fundamental for infant growth and development ${ }^{(37)}$, a major decrease in blood lipids could have been harmful. It is to be noted that including LF19 in the weaning diet posed no safety issues. In relation to long-term lipid-lowering effects of breastfeeding, adult TC and LDL concentrations are marginally lower ${ }^{(5)}$. No study has thus far been able to convincingly explain the mechanisms behind this. One plausible factor may be via long-lasting effects on gut microbiota establishment and composition in breastfed infants.

We found significantly higher levels of putrescine in the LF19 compared with the placebo group. Putrescine is a polyamine involved in cell growth and differentiation ${ }^{(38,39)}$. Dietary and gut bacteria-derived polyamines contribute to the total polyamine body pool $^{(40)}$. Polyamines are present in breast milk and have been proposed to be important in neonatal gut maturation ${ }^{(41)}$. Some probiotic strains increase gut integrity ${ }^{(42)}$ and the present findings suggest that one possible mechanism might be through increased polyamine levels.

The model loadings revealed a decreased pattern of metabolites linked to the pentose phosphate pathway in the LF19 group, with the opposite trend in the placebo group. The pentose phosphate pathway has mainly two metabolic functions: to meet the need of NADPH for reductive biosynthesis, such as fatty acids, cholesterol, neurotransmitters and nucleotides and in formation of ribose, a component of essential biomolecules 
such as ATP, DNA and RNA ${ }^{(43)}$. The decreased pattern could be a result of lower demand for reductive agents and/or nucleotides. In fact, some lactobacilli possess antioxidative qualities and are able to decrease accumulation of reactive oxygen species ${ }^{(44,45)}$. In combination with the increase in putrescine, which also protects cells from oxidative stress ${ }^{(46)}$, this might imply antioxidative qualities of LF19, but needs further validation.

We observed age-related changes, with a significant increase in PA from 5.5 to 13 months of age. PA is most likely a metabolite of lysine catabolism. It has an important depressive role in the central nervous system ${ }^{(47)}$ and acts as a precursor of microbial secondary metabolites ${ }^{(48)}$. It is suggested to be a derivate from intestinal bacteria rather than from $\operatorname{diet}^{(47)}$, and we speculate that the increase in PA reflects increasing gut microbial diversity during weaning ${ }^{(49)}$. Several amino acids increased during the second half of infancy, possibly reflecting a higher dietary protein intake, as complementary foods were successively introduced $^{(50)}$. Studies on plasma amino acid patterns during weaning are sparse, but data suggest an increase with age ${ }^{(51,52)}$.

The combined metabolomics and chemometric bioinformatics approach allowed us to model and interpret the complex metabolic interactions with and without the influence of LF19, and with increasing age. The benefit of the methodology was clearly highlighted when studying age-related changes in treatment groups, where we could extract differentiating metabolite patterns by making comparisons of calculated model loadings (variable weights). Differences in individual metabolites were not detected when comparing treatment groups in a separate model, but were detected using univariate statistics. This highlights the importance of using multivariate and univariate statistics in concert not to miss relevant metabolic changes. In addition, the multivariate models helped to reveal individual (low-to-intermediate or high) metabolic responses, irrespective of LF19 treatment. This underlines that effects of probiotics vary. The presented approach could be valuable in dose-response studies, and ultimately in tailoring effective treatment and prevention strategies with probiotics.

A limitation of the present study is that we only recorded daily intake of weaning cereals and breastfeeding, while other food intakes were not registered. However, as the two compared groups were randomly chosen and showed no differences in growth, we assume that the influence of possible differences in dietary intakes were not substantial at the group level.

In summary, we show that feeding LF19 during weaning affects the metabolic profile. We observed a significant decrease of palmitic (c16:0) and palmitoleic acid (c16:1) and an increase in the polyamine putrescine in the LF19 compared with the placebo group. Whether the observed decrease of palmitoleic acid (c16:1), a major MUFA which was previously shown to be strongly linked to visceral obesity, and the increase of putrescine, a polyamine with importance for gut integrity, will be associated with long-term health consequences are being followed.

\section{Acknowledgements}

We acknowledge the participating families; Margareta Henriksson, RuthGerd Larsson and Margareta Bäckman for invaluable contact with parents, and their infants and excellent assistance with the administration of trial products and blood samplings; and associate professor Hans Stenlund for invaluable advice on statistical procedures. C. E. W. has received funding and speaker honorarium from Arla Foods. O. H. has received funding from Semper $\mathrm{AB}$ and Arla Foods. The other authors declare no conflict of interest. The present study was supported by Semper AB, Sweden, Swedish Research Council for Environment, Agricultural Sciences and Spatial Planning (FORMAS); Swedish Agency for Innovation Systems (VINNOVA); Västerbotten county council (ALF); Ronald McDonald Fund; Swedish Nutrition Foundation and Oskar-fonden; and European Union's Seventh Framework Programme under grant agreement no. 222720. C. E. W. is sponsored by a fellowship from the Swedish Society of Medical Research. Semper AB personnel were involved in discussions regarding study design. Sponsors had no involvement in the collection, analysis and interpretation of data, in writing the report or in the decision to submit the paper for publication. E. C. and H. A. performed all metabolomics and multivaraiate analysis. F. K. V. and C. E. W. performed some statistical analysis. E. C., F. K. V., H. A. and C. E. W. wrote the manuscript. O. H. contributed to the discussions. C. E. W. and $\mathrm{O} . \mathrm{H}$. were responsible for the design of the project.

\section{References}

1. Vickers M (2011) Developmental programming of the metabolic syndrome - critical windows for intervention. World J Diabetes 2, 137-148.

2. Ogden CL, Carroll MD, Kit BK, et al. (2012) Prevalence of obesity and trends in body mass index among US children and adolescents, 1999-2010. JAMA 307, 483-490.

3. Huh SY, Rifas-Shiman SL, Taveras EM, et al. (2011) Timing of solid food introduction and risk of obesity in preschool-aged children. Pediatrics 127, E544-E551.

4. Moorcroft KE, Marshall JL \& McCormick FM (2011) Association between timing of introducing solid foods and obesity in infancy and childhood: a systematic review. Mater Child Nutr 7, 3-26.

5. Owen CG, Whincup PH, Kaye SJ, et al. (2008) Does initial breastfeeding lead to lower blood cholesterol in adult life? A quantitative review of the evidence. Am JClin Nutr 88, 305-314.

6. Delzenne NM, Neyrinck AM, Bäckhed F, et al. (2011) Targeting gut microbiota in obesity: effects of prebiotics and probiotics. Nat Rev Endocrinol 7, 639-646.

7. Adlerberth I \& Wold AE (2009) Establishment of the gut microbiota in Western infants. Acta Paediatr 98, 229-238.

8. Delzenne NM \& Cani PD (2011) Interaction between obesity and the gut microbiota: relevance in nutrition. Annu Rev Nutr 31, 15-31.

9. Ley RE, Bäckhed F, Turnbaugh P, et al. (2005) Obesity alters gut microbial ecology. Proc Natl Acad Sci U S A 102, 11070-11075.

10. Kalliomäki M, Salminen S \& Isolauri E (2008) Positive interactions with the microbiota: probiotics. Adv Exp Med Biol 635, 57-66.

11. Turnbaugh PJ, Ley RE, Mahowald MA, et al. (2006) An obesity-associated gut microbiome with increased capacity for energy harvest. Nature 444, 1027-1031.

12. Turnbaugh PJ, Ridaura VK, Faith JJ, et al. (2009) The effect of diet on the human gut microbiome: a metagenomic analysis in humanized gnotobiotic mice. Sci Trans Med 1, Gra14. 
13. Kalliomäki M, Collado MC, Salminen S, et al. (2008) Early differences in fecal microbiota composition in children may predict overweight. Am J Clin Nutr 87, 534-538.

14. FAO/WHO (2001) Health and Nutritional Properties of Probiotics in Food including Powder Milk with Live Lactic Acid Bacteria. Report of a Joint FAO/WHO Expert Consultation on Evaluation of Health and Nutritional Properties of Probiotics in Food Including Powder Milk with Live Lactic Acid Bacteria Cordoba, Argentina ftp.fao.org/es/esn/food/probio report_en.pdf.

15. Laitinen K, Collado M \& Isolauri E (2010) Early nutritional environment: focus on health effects of microbiota and probiotics. Benef Microbes 1, 383-390.

16. Ooi LG \& Liong MT (2010) Cholesterol-lowering effects of probiotics and prebiotics: a review of in vivo and in vitro findings. Int J Mol Sci 11, 2499-2522.

17. Nerstedt A, Nilsson EC, Ohlson K, et al. (2007) Administration of Lactobacillus evokes coordinated changes in the intestinal expression profile of genes regulating energy homeostasis and immune phenotype in mice. Br J Nutr 97 $1117-1127$

18. Aronsson L, Huang Y, Parini P, et al. (2010) Decreased fat storage by Lactobacillus paracasei is associated with increased levels of angiopoietin-like 4 protein (ANGPTL4). PLoS One 5, e13087.

19. Fiehn O (2002) Metabolomics - the link between genotypes and phenotypes. Plant Mol Biol 48, 155-171.

20. Kell DB (2004) Metabolomics and systems biology: making sense of the soup. Curr Opin Microbiol 7, 296-307.

21. Wang ZN, Klipfell E, Bennett BJ, et al. (2011) Gut flora metabolism of phosphatidylcholine promotes cardiovascular disease. Nature 472, 57-63.

22. Brennan L (2008) Metabolomic applications in nutritional research. Proc Nutr Soc 67, 404-408.

23. West CE, Gothefors L, Granström M, et al. (2008) Effects of feeding probiotics during weaning on infections and antibody responses to diphtheria, tetanus and Hib vaccines. Pediatr Allergy Immunol 19, 53-60.

24. The International Response to Helsinki V (2000) The WMA's Declaration of Helsinki on Ethical Principles for Medical Research Involving Human Subjects, As Adopted by the 52nd WMA General Assembly. Edinburgh. www.wma.net/en/20 activities/10ethics/10helsinki/intl_response_helsinki.pdf

25. Friedewald WT, Levy RI \& Fredrickson DS (1972) Estimation of the concentration of low-density lipoprotein cholesterol in plasma, without use of the preparative ultracentrifuge. Clin Chem 18, 499-502.

26. Wishart DS, Tzur D, Knox C, et al. (2007) HMDB: the human metabolome database. Nucleic Acids Res 35, D521-D526.

27. Wiklund S, Johansson E, Sjöström L, et al. (2008) Visualization of GC/TOF-MS-based metabolomics data for identification of biochemically interesting compounds using OPLS class models. Anal Chem 80, 115-122.

28. Kunesova M, Hainer V, Tvrzicka E, et al. (2002) Assessment of dietary and genetic factors influencing serum and adipose fatty acid composition in obese female identical twins. Lipids 37, 27-32.

29. Paillard F, Catheline D, Le Duff F, et al. (2008) Plasma palmitoleic acid, a product of stearoyl-coA desaturase activity, is an independent marker of triglyceridemia and abdominal adiposity. Nutr Metab Cardiovasc Dis 18, 436-440.

30. Okada T, Furuhashi N, Kuromori Y, et al. (2005) Plasma palmitoleic acid content and obesity in children. Am J Clin Nutr 82, 747-750.
31. Lands WE (1995) Long-term fat intake and biomarkers. Am J Clin Nutr 61, S721-S725.

32. Sen B, Bose K, Shaikh S, et al. (2010) Prediction equations for body-fat percentage in Indian infants and young children using skinfold thickness and mid-arm circumference. $J$ Health Popul Nutr 28, 221-229.

33. Guo Z, Liu XM, Zhang QX, et al. (2011) Influence of consumption of probiotics on the plasma lipid profile: a metaanalysis of randomised controlled trials. Nutr Metab Cardiovasc Dis 21, 844-850

34. Norin E \& Midtvedt T (2006) In born germfree - microbioal dependent. In Gastrointestinal Microbiology, pp. 273-284 [AC Ouwehand and EE Vaughan, editors]. New York, NY: Taylor \& Francis

35. Edwards CA \& Parrett AM (2002) Intestinal flora during the first months of life: new perspectives. BrJ Nutr 88, S11-S18.

36. Alliet P, Scholtens P, Raes M, et al. (2007) Effect of prebiotic galacto-oligosaccharide, long-chain fructo-oligosaccharide infant formula on serum cholesterol and triacylglycerol levels. Nutrition 23, 719-723.

37. Uauy R \& Dangour AD (2009) Fat and fatty acid requirements and recommendations for infants of $0-2$ years and children of 2-18 years. Ann Nutr Metab 55, 76-96.

38. McCormack SA \& Johnson LR (1991) Role of polyamines in gastrointestinal mucosal growth. Am J Physiol 260, G795-G806.

39. Pegg AE (2009) Mammalian polyamine metabolism and function. IUBMB Life 61, 880-894.

40. Löser C (2000) Polyamines in human and animal milk. Br J Nutr 84, S55-S58.

41. Larqué E, Sabater-Molina M \& Zamora S (2007) Biological significance of dietary polyamines. Nutrition 23, 87-95.

42. Rosenfeldt V, Benfeldt E, Valerius NH, et al. (2004) Effect of probiotics on gastrointestinal symptoms and small intestinal permeability in children with atopic dermatitis. I Pediatr 145, 612-616.

43. Berg JT, Tymoczko JL \& Stryer L (2002) Biochemistry, 5th ed., pp. 563-568 [S Moran, editor]. New York, NY: W.H. Freeman.

44. Kullisaar T, Zilmer M, Mikelsaar M, et al. (2002) Two antioxidative lactobacilli strains as promising probiotics. Int J Food Microbiol 72, 215-224.

45. van de Guchte M, Penaud S, Grimaldi C, et al. (2006) The complete genome sequence of Lactobacillus bulgaricus reveals extensive and ongoing reductive evolution. Proc Natl Acad Sci U S A 103, 9274-9279.

46. Chattopadhyay MK, Tabor CW \& Tabor H (2003) Polyamines protect Escherichia coli cells from the toxic effect of oxygen. Proc Natl Acad Sci U S A 100, 2261-2265.

47. Fujita T, Fujita M, Kodama T, et al. (2003) Determination of Dand L-pipecolic acid in food samples including processed foods. Ann Nutr Metab 47, 165-169.

48. He M (2006) Pipecolic acid in microbes: biosynthetic routes and enzymes. J Ind Microbiol Biotechnol 33, 401-407.

49. Eckburg PB, Bik EM, Bernstein CN, et al. (2005) Diversity of the human intestinal microbial flora. Science 308, 1635-1638.

50. Lind T, Hernell O, Lönnerdal B, et al. (2004) Dietary iron intake is positively associated with hemoglobin concentration during infancy but not during the second year of life. J Nutr 134, 1064-1070.

51. Hammarqvist F, Angsten G, Meurling S, et al. (2010) Agerelated changes of muscle and plasma amino acids in healthy children. Amino Acids 39, 359-366.

52. Lepage N, McDonald N, Dallaire L, et al. (1997) Age-specific distribution of plasma amino acid concentrations in a healthy pediatric population. Clin Chem 43, 2397-2402. 